\title{
Nanomole per Liter per Minute
}

National Cancer Institute

\section{Source}

National Cancer Institute. Nanomole per Liter per Minute. NCI Thesaurus. Code

C122219.

A rate unit equal to the number of nanomoles per unit of volume equal to one liter per unit of time equal to one minute. 